Experimental results for 2D magnetic resonance electrical impedance tomography (MR-EIT) using magnetic flux density in one direction

This content has been downloaded from IOPscience. Please scroll down to see the full text.

2003 Phys. Med. Biol. 483485

(http://iopscience.iop.org/0031-9155/48/21/003)

View the table of contents for this issue, or go to the journal homepage for more

Download details:

IP Address: 139.179.72.198

This content was downloaded on 29/08/2017 at 13:49

Please note that terms and conditions apply.

You may also be interested in:

Equipotential projection for MR-EIT

Mahir S Özdemir, B Murat Eyübolu and Orçun Özbek

CCVSR algorithm for MR-EIT

Özlem Birgül, B Murat Eyüboglu and Y Ziya Ider

Anisotropic conductivity imaging with MREIT

Evren Deirmenci and B Murat Eyübolu

MREIT using low amplitude current

Ozlem Birgul, Mark J Hamamura, L Tugan Muftuler et al.

MREIT imaging of head conductivity

Nuo Gao, S A Zhu and Bin He

Algebraic reconstruction for 3D MREIT

Y Ziya Ider and Serkan Onart

Harmonic Bz algorithm in MREIT

Suk Hoon Oh, Byung II Lee, Eung Je Woo et al.

FT-MRCDI from one component of magnetic flux density Yusuf Ziya Ider, Ozlem Birgul, Omer Faruk Oran et al.

FILL IN THE MISSING PIECES.
When every piece counts, get the whole picture with LinacView and Adaptivo.
VISIT US AT ASTRO BOOTH 2537 STANDARDIMAGING. 


\title{
Experimental results for 2D magnetic resonance electrical impedance tomography (MR-EIT) using magnetic flux density in one direction
}

\author{
Özlem Birgüil ${ }^{1,3}$, B Murat Eyüboğlu ${ }^{1}$ and Y Ziya İder ${ }^{2}$ \\ ${ }^{1}$ Department of Electrical and Electronics Engineering, Middle East Technical University, \\ 06531 Ankara, Turkey \\ ${ }^{2}$ Department of Electrical and Electronics Engineering, Bilkent University, 06800 Ankara, \\ Turkey \\ E-mail: meyub@metu.edu.tr
}

Received 22 April 2003, in final form 29 July 2003

Published 10 October 2003

Online at stacks.iop.org/PMB/48/3485

\begin{abstract}
Magnetic resonance electrical impedance tomography (MR-EIT) is an emerging imaging technique that reconstructs conductivity images using magnetic flux density measurements acquired employing MRI together with conventional EIT measurements. In this study, experimental MR-EIT images from phantoms with conducting and insulator objects are presented. The technique is implemented using the 0.15 T Middle East Technical University MRI system. The dc current method used in magnetic resonance current density imaging is adopted. A reconstruction algorithm based on the sensitivity matrix relation between conductivity and only one component of magnetic flux distribution is used. Therefore, the requirement for object rotation is eliminated. Once the relative conductivity distribution is found, it is scaled using the peripheral voltage measurements to obtain the absolute conductivity distribution. Images of several insulator and conductor objects in saline filled phantoms are reconstructed. The L2 norm of relative error in conductivity values is found to be $13 \%, 17 \%$ and $14 \%$ for three different conductivity distributions.
\end{abstract}

(Some figures in this article are in colour only in the electronic version)

\section{Introduction}

Magnetic resonance electrical impedance tomography (MR-EIT) is an emerging imaging modality that reconstructs electrical conductivity in a volume conductor using internal

3 Currently at: Tu and Yuen Center for Functional Onco-Imaging, University of California, Irvine, CA, USA. 


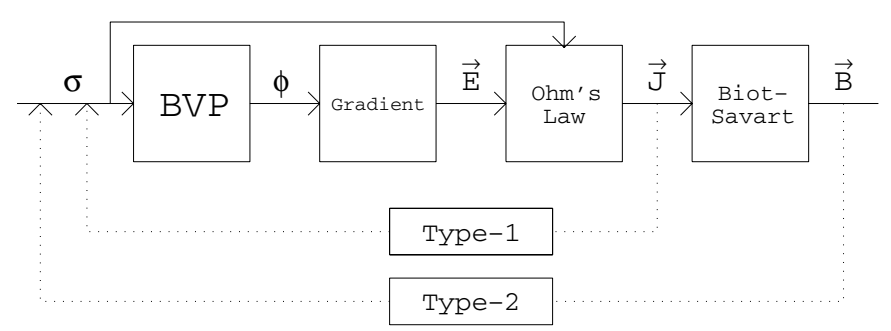

Figure 1. System variables in the MR-EIT forward problem and illustration of type 1 and type 2 reconstruction algorithms: BVP represents the related boundary value problem, $\sigma$ is the conductivity, $\phi$ is the potential field, $\vec{E}$ is the electric field, $\vec{J}$ is the current density and $\vec{B}$ is the magnetic flux density. Solid lines show information flow in the forward problem and dotted lines show information flow in type 1 and type 2 reconstruction algorithms.

magnetic flux density and, optionally, peripheral voltage measurements (Zhang 1992, Woo et al 1994, Birgül and İder 1995, İder and Birgül 1998, Birgül et al 1999, 2001a, Eyüboğlu et al 2002, Kwon et al 2002a, 2002b, Khang et al 2002, Birgül 2002). A detailed summary of previous work done on MR-EIT is given by Birgül et al (2003). High resolution conductivity images can be reconstructed by MR-EIT. The spatial resolution is ultimately limited by the resolution of magnetic resonance imaging (MRI) system. In practice, the spatial resolution will be determined by the SNR of the measurements.

Based on the data used in image reconstruction, MR-EIT reconstruction algorithms can be classified into two groups, type 1 and type 2 . In figure 1 , the relation between system variables is explained in order to illustrate the basis of this classification. For a given object with conductivity distribution $\sigma$, as a result of applied potentials and/or injected currents on the boundary of the object, potential and electric field distributions are generated in the object. The current distribution in the object is given by $\vec{J}=\sigma \vec{E}$. The internal current distribution, $\vec{J}$, sets up a magnetic flux density distribution, $\vec{B}$, both inside and outside the object. Using MRI, $\vec{B}$ inside the object can be measured (Scott et al 1991). Using the measured $\vec{B}$, one can find $\vec{J}$ from $\vec{J}=(\nabla \times \vec{B}) / \mu_{0}$. In type 1 reconstruction algorithms, $\vec{J}$ is used as data whereas in type 2 algorithms, $\vec{B}$ is used as data. The algorithms proposed by Zhang (1992), Birgül et al (2001a), Eyüboğlu et al (2002) and Kwon et al (2002b) are examples of type 1 reconstruction algorithms. The sensitivity matrix approach of Birgül and İder (1995) and İder and Birgül (1998) is an example of type 2 image reconstruction algorithm.

The major disadvantage of type 1 algorithms is that in order to find $\vec{J}$ from $\vec{J}=$ $(\nabla \times \vec{B}) / \mu_{0}$, one needs to measure all three components of $\vec{B}$ using MRI. Since only the $z$-component (where $z$ is the direction of the main magnetic field of MR system's main magnet) of $\vec{B}$ can be measured by magnetic resonance, the object must be rotated, in order to acquire all three components of $\vec{B}$. This is a severe limitation experimentally. It may also be possible to rotate the main magnetic field of the MRI magnet by selectively exciting different coils (Birgül et al 2003), but present MRI systems do not have this capability. In type 2 algorithms on the other hand, there is the opportunity of using only $B_{z}$ to reconstruct conductivity.

Uniqueness conditions for type 1 algorithms are already established. At least two current injection profiles yielding two current densities such that $\left|\vec{J}_{1} \times \vec{J}_{2}\right| \neq 0$ are required for unique image reconstruction (Kwon et al 2002b, İder et al 2003). On the other hand uniqueness conditions for type 2 algorithms utilizing only $B_{z}$ are not established.

With type 1 , it is possible to reconstruct a relative conductivity distribution using $\vec{J}$ alone. By relative, it is meant that $\sigma$ is reconstructed apart from a multiplying constant. It has been 
shown that measurement of at least one voltage difference at the boundary is sufficient to find this multiplying constant (Kwon et al 2002b).

In this paper, experimental results acquired with the $0.15 \mathrm{~T}$ Middle East Technical University (METU) MRI system are presented. The image reconstruction algorithm used is a modified version of the sensitivity matrix reconstruction algorithm proposed by Birgül and İder (1995) and İder and Birgül (1998). The most attractive feature of this algorithm is that only one component of the magnetic flux density measurement, $B_{z}$, at a single slice is sufficient for conductivity reconstruction. Hence, the necessity of rotation of the object within the magnet is eliminated. Although the sensitivity matrix approach was previously used in İder and Birgül (1998), in that study (i) only one ac current injection pattern was used, (ii) peripheral voltage measurements were not included and therefore relative images were reconstructed, and (iii) ac current at 100-200 Hz was used and magnetic flux density was measured with very low resolution due to the limitations of the measurement technique used (İder and Müftüler 1997). The major novel element of this study is the experimental implementation of the use of only one component of magnetic flux density measurement at a single slice, thus eliminating the need for the rotation of the object. In addition, two current injection patterns and peripheral voltage measurements are included. A well established technique for $B_{z}$ measurement is used (Scott et al 1991). Elimination of the effect of current carrying wires is also included in image reconstruction.

Results based on real measurements as opposed to simulations are scarce in the MR-EIT literature (İder and Birgül 1998, Khang et al 2002, Birgül et al 2001a). This study also fills this gap by providing several experimental results from various $2 \mathrm{D}$ conductivity distributions.

Accurate conductivity images can be used in monitoring physiological functions (Frerichs 2000, Mueller et al 2001), and detecting cancerous tissues (Piperno et al 1990, Cherepenin et al 2001) or solution of bioelectric field problems such as source localization (Gonçalves et al 2000, Eyüboğlu and Özbek 2003).

In the next section, the MR-EIT problem and the experimental phantom used in this study are explained. In section 3, the proposed image reconstruction algorithm is derived. Relative and absolute conductivity images reconstructed using experimental data are presented in section 4.

\section{Experimental set-up}

Figure 2 shows the volume conductor phantom and the locations of the electrodes for current injection and voltage measurements. The thickness of the phantom is small compared to the other two dimensions and the problem is formulated in two dimensions as an approximation. The phantom is filled with $9 \mathrm{~g} / 1 \mathrm{NaCl}, 1 \mathrm{~g} / 1 \mathrm{CuSO}_{4} \cdot 5 \mathrm{H}_{2} \mathrm{O}$ solution. This solution will be referred to as saline hereafter. Using a four-electrode conductivity measurement cell (Baker 1989), the conductivity of the saline is measured as $20 \mathrm{mS} \mathrm{cm}^{-1}$. In order to generate different conductivity distributions, insulator and/or conductor objects are placed inside the saline filled phantom and the side cover is sealed with silicon sealant. Although the insulator shells are filled with the same solution to have MR signal, inner and outer solutions are not electrically connected, thus the region surrounded by the shell acts as an electrical insulator. In order to generate electrically connected regions with different conductivity values, objects formed using synthetic sponge with uniform pore structure are inserted into the phantom. It is ensured that the saline solution fills the pores of the sponge completely and no air bubbles are left inside the sponge. The conductivity value of the sponge is measured approximately to be around $10 \mathrm{mS} \mathrm{cm}{ }^{-1}$ by the four-electrode conductivity measurement cell. In the four-electrode conductivity measurement cell, the sponge fits exactly into the cell. However, in some of our 


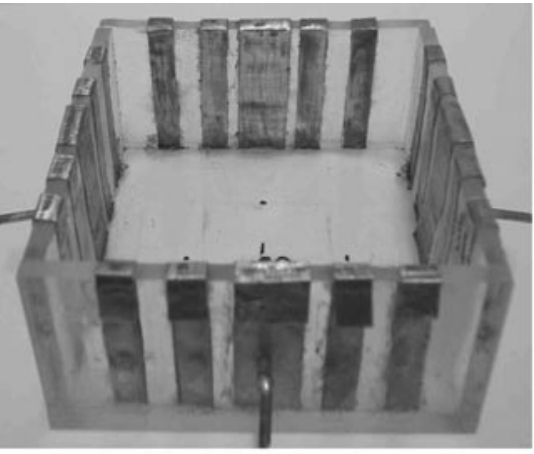

(a)

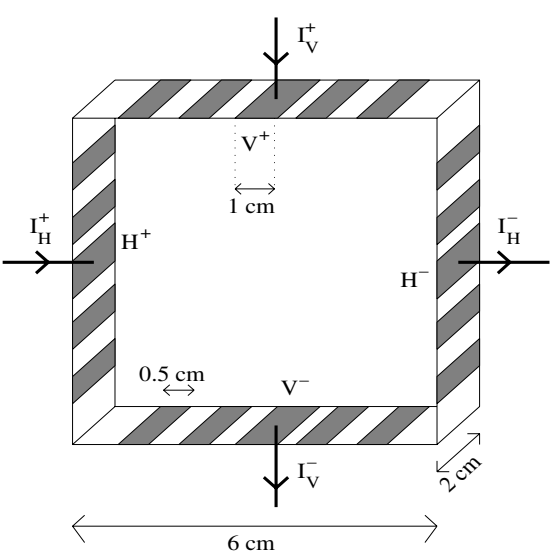

(b)

Figure 2. The square volume conductor phantom and electrodes. (a) Picture of the phantom with side cover open to show a better view of the electrodes, and (b) diagram and dimensions. The shaded regions are the electrodes.

phantoms, the sponge objects can expand freely. In such cases, the sponge region is expected to have conductivity higher than the measured value in the conductivity cell.

There are five electrodes at each side of the square phantom making a total of 20 electrodes (figure 2). The current is injected using opposite pairs at the centre of each side giving two different current injection profiles. Electrodes $\mathrm{H}^{+}$and $\mathrm{H}^{-}$are used for horizontal and $\mathrm{V}^{+}$and $\mathrm{V}^{-}$are used for vertical current injection cases. The peripheral voltage values are measured from the rest of the electrodes using adjacent pairs. Current injection electrodes are not used for voltage measurements. A total of 30 independent voltage measurements are recorded for two current injection cases.

Magnetic flux density is extracted from the MR phase images acquired during injection of bipolar dc current pulses (Scott et al 1991). Two magnetic flux density distributions that correspond to horizontal and vertical current injection cases are measured. Note that for both horizontal and vertical cases, the component of the magnetic flux density parallel to the main MR field, which is in the $z$-direction, is measured.

It is not trivial to obtain the phase term $\phi(x, y)$ for a complex image, in general. $\phi(x, y)$ is uniquely defined only in the principal value range of $(-\pi, \pi]$ and any phase value outside this range is wrapped to this interval resulting in the wrapped phase image, $\hat{\phi}(x, y)$. In this study, Liang's model-based method for phase unwrapping is implemented (Liang 1996). In this method, the unwrapped phase function is represented as the sum of a truncated Taylor series and a residual function and the unwrapping problem is converted to a parameter estimation problem.

Placement of current carrying wires in the magnet is an important issue in current density imaging (Gamba and Delpy 1997). Either the wires must be placed so that currents through the wires would not generate magnetic flux density in the direction of the main field or their contributions must be included in the calculations if their effect cannot be eliminated totally. In our set-up, the $z$-direction is the MR system's main field direction and the object is placed on the $x-y$ plane. In order to avoid the effect of current carrying wires, the wires must be placed parallel to the $z$-axis. Although in our experimental studies, wires are placed along the $z$-axis, because of the bending of the wires at phantom connections, an additional field is observed 
in the measured magnetic flux density. To eliminate the effect of current carrying wires, the magnetic flux density generated due to current through the bending wires is calculated using the Biot-Savart law, then the calculated flux density is subtracted from the experimentally measured flux density. The amount of improvement achieved using this approach is discussed in the discussion section 5 .

\section{Sensitivity-based image reconstruction algorithm}

In this section, first the forward transformation from conductivity to magnetic flux density is derived. Then, for image reconstruction (i.e. for the solution of the inverse problem) an iterative algorithm, which is based on linearizing the forward transformation at each step, is presented.

\subsection{Forward transformation from conductivity to magnetic flux density}

Calculations of surface potential values and inner magnetic flux density distribution for a known conductivity distribution and boundary conditions are called the forward problem of MR-EIT. Solution of the forward problem is explained for the two-dimensional case. In fact, as explained in section 2.1, the experimental phantoms used in this study mimic the $2 \mathrm{D}$ case since the electrodes cover the entire thickness of the phantom and the inside conductivity is constant along the thickness. The relation between conductivity and potential distributions in the object is given by Laplace's equation,

$$
\nabla(\sigma(x, y) \nabla \phi(x, y))=0 \quad(x, y) \in S
$$

where $\sigma(x, y)$ is electrical conductivity distribution, $\phi(x, y)$ is the electrical potential distribution and $S$ is the domain representing the object to be imaged. For the case of current injected on the surface via two current electrodes, the Neumann boundary condition holds, which is

$$
\sigma(x, y) \frac{\partial \phi(x, y)}{\partial n}=\left\{\begin{array}{cl}
j & \text { on positive current electrode } \\
-j & \text { on negative current electrode } \\
0 & \text { elsewhere }
\end{array}\right.
$$

where $j$ is the magnitude of the applied current density and $\partial \phi(x, y) / \partial n$ is the change in electrical potential in the direction of the outward normal.

In order to calculate the magnetic flux density distribution, $\vec{B}(x, y)$, inside the object, current density distribution, $\vec{J}(x, y)$, must be calculated first. Since the conductivity is assumed to be known in the forward problem, electric field intensity distribution, $\vec{E}(x, y)$, and the corresponding $\vec{J}(x, y)$ are found using

$$
\vec{E}(x, y)=-\nabla \phi(x, y)
$$

and

$$
\vec{J}(x, y)=\sigma(x, y) \vec{E}(x, y) .
$$

Finally, $\vec{B}(x, y)$ is calculated using the Biot-Savart relation,

$$
\vec{B}(x, y)=\frac{\mu_{0}}{4 \pi} \int \frac{\vec{J}(x, y) \mathrm{d} S \times \hat{a}_{R}}{R^{2}},
$$

where $\vec{B}(x, y)$ is the magnetic flux density at measurement points, $\mu_{0}$ is the permeability of free space, $R$ is the distance between source and field points, and $\hat{a}_{R}$ is the unit vector pointing 
from source, $\left(x^{\prime}, y^{\prime}\right)$, to the field point, $(x, y)$. Only the $z$-component of the magnetic flux density is used in image reconstruction and is given by

$$
B_{z}(x, y)=\frac{\mu_{0}}{4 \pi} \int \frac{\left(J_{x}\left(x^{\prime}, y^{\prime}\right)\right)\left(y-y^{\prime}\right)-\left(J_{y}\left(x^{\prime}, y^{\prime}\right)\right)\left(x-x^{\prime}\right)}{R^{3}} \mathrm{~d} S .
$$

Finite element method (FEM) is used to solve equation (1) (Silvester and Ferrari 1996). First order triangular elements are used to discretize the imaging region, $S$. Each element is assumed to have uniform conductivity, and the potential distribution in each element is approximated by a plane. Consequently, the electric field and current density inside each element are constant. In the calculation of magnetic flux density from current density using the Biot-Savart relation, each finite element is taken to act as a current source located at its centre of gravity, and the field points are also selected as the element centres. Although magnetic flux values are calculated at triangular element centres, in practice magnetic flux density is measured on a rectangular grid, using MRI. Two triangular elements in FEM mesh correspond to one square pixel in the MRI images. In order to match the calculated and the measured field points, the magnetic flux density at the centre of a square pixel is found by averaging the values at the centres of two corresponding triangles. Note that a singularity is encountered when source and field points are the same. To avoid this, the contribution of each element on itself is assumed to be zero. Although this introduces some errors in field calculations, the effect is minimal when the average of two triangles is assigned to a square pixel. For uniform current density and uniform conductivity distribution in a rectangular $2 \mathrm{D}$ object, the Biot-Savart integral is evaluated analytically and compared with numerical results for each pixel to understand the amount of error introduced due to this approximation. Maximum and mean pixel errors in magnetic flux density are calculated to be $2 \%$ and $0.9 \%$, respectively.

This formulation assumes that all current flows in the mid-plane (along the thickness) of the phantom. We have also explored the effect of the finite thickness of the phantom using a 3D forward formulation as will be discussed in section 5.3.

Given that there are $n$ finite elements and $m$ measurement points, the $(n \times 1)$ vector $\sigma$ is used to represent the element conductivities, and the $(m \times 1)$ vector $\mathbf{b}$ is used to represent the measured $z$-component magnetic flux density values. The forward relation between conductivity and $z$-component magnetic flux density is non-linear, i.e.

$$
\mathbf{b}=\mathbf{F}(\sigma)
$$

where $\mathbf{F}$ is a non-linear function of $\boldsymbol{\sigma}$.

If data are collected for two injected current patterns, then two forward transformations $\mathbf{F}_{1}$ and $\mathbf{F}_{2}$ are obtained for the two measurement vectors $\mathbf{b}_{1}$ and $\mathbf{b}_{2}$. The two systems of equations can then be combined to obtain the combined forward transformation

$$
\mathbf{b}_{c}=\mathbf{F}_{c}(\sigma)
$$

where $\mathbf{F}_{c}=\left[\begin{array}{l}\mathbf{F}_{1} \\ \mathbf{F}_{2}\end{array}\right]$ and $\mathbf{b}_{c}=\left[\begin{array}{l}\mathbf{b}_{1} \\ \mathbf{b}_{2}\end{array}\right]$ are the combined forward transformation and combined measurement vector, respectively.

\subsection{Linearization of the forward transformation}

The forward transformation can be linearized around an initial conductivity vector $\sigma_{\text {ini }}$. The relation between change in conductivity, $\Delta \sigma$, and change in magnetic flux density, $\Delta \mathbf{b}=\left(\mathbf{b}-\mathbf{b}_{\text {ini }}\right)$, where $\mathbf{b}_{\text {ini }}$ is the magnetic flux density corresponding to $\sigma_{\text {ini }}$, can then be written in matrix form as

$$
\Delta \mathbf{b}=\mathbf{S} \Delta \sigma
$$


where $\mathbf{S}$ is the $(m \times n)$ sensitivity matrix which is in fact the Jacobian of $\mathbf{F}$. S represents the forward transformation from the conductivity perturbation domain to the magnetic flux perturbation domain. The sensitivity matrix can be obtained either numerically or semianalytically. In the numerical approach, the conductivities of the elements are perturbed one at a time, and the calculated vector of perturbation in magnetic flux density values forms the corresponding column of the sensitivity matrix. In the semianalytical approach, a mathematical expression for the entries of the sensitivity matrix is derived using linearization (Birgül 1997). Using vector identities and neglecting second order variations, in the continuous domain, the mathematical expression between $\Delta \vec{B}_{z}$ and $\Delta \sigma$ is obtained to be

$\Delta \vec{B}_{z}=-\frac{\mu_{0}}{4 \pi} \int_{S^{\prime}} \frac{\vec{R} \times \nabla \phi_{\text {ini }}}{R^{3}} \Delta \sigma \mathrm{d} S^{\prime}-\frac{\mu_{0}}{4 \pi} \int_{S^{\prime}} \sigma_{\text {ini }} \frac{\vec{R} \times(\partial(\nabla \phi) / \partial \sigma)}{R^{3}} \Delta \sigma \mathrm{d} S^{\prime}$

where $\phi_{\text {ini }}$ is the potential distribution for the initial conductivity distribution, $\nabla \phi_{\text {ini }}$ is its gradient, $\vec{R}$ is the vector from the source point to the field point, $\mu_{0}$ is the permeability of free space and $\mathrm{d} S^{\prime}$ is the differential surface element in the imaging region, $S^{\prime} . \nabla \phi_{\text {ini }}$ is calculated using the FEM solver, and therefore, calculation of $\mathbf{S}$ using this approach is classified as semianalytical. The term giving the sensitivity relation between conductivity and voltage measurements, $\partial(\nabla \phi) / \partial \sigma$, around the given initial $\sigma_{\text {ini }}(x, y)$, is also calculated semianalytically as explained in Birgül (1997).

If data are collected for two injected current patterns, then two sensitivity matrices, $\mathbf{S}_{1}$ and $\mathbf{S}_{2}$, are obtained for the two measurement vectors $\mathbf{b}_{1}$ and $\mathbf{b}_{2}$. The two systems of equations can then be combined to obtain the combined sensitivity matrix equation

$$
\Delta \mathbf{b}_{c}=\mathbf{S}_{c} \Delta \boldsymbol{\sigma}
$$

where $\mathbf{S}_{c}=\left[\begin{array}{c}\mathbf{S}_{1} \\ \mathbf{S}_{2}\end{array}\right]$ is the combined sensitivity matrix and $\Delta \mathbf{b}_{c}=\left[\begin{array}{l}\mathbf{b}_{1}-\mathbf{b}_{1 \text { ini }} \\ \mathbf{b}_{2}-\mathbf{b}_{2 \mathrm{ini}}\end{array}\right]$ is the combined change in magnetic flux density.

\subsection{Image reconstruction algorithm}

Equation (11) must be solved at each iteration of the iterative reconstruction algorithm explained below. In order to handle cases of noisy measurements, truncated singular value decomposition (SVD) is used. In SVD, the combined sensitivity matrix is written as the multiplication of three matrices as:

$$
\mathbf{S}_{c}=\mathbf{U} \Lambda \mathbf{V}^{T}
$$

where $\mathbf{U}$ and $\mathbf{V}$ are orthonormal matrices whose columns are the measurement and image basis vectors respectively and $\Lambda$ is a diagonal matrix with entries $\lambda_{i}$, being the $i$ th singular value. The inverses of $\mathbf{U}$ and $\mathbf{V}$ matrices are equal to the transposes of themselves due to orthonormality, and the inverse of the diagonal matrix $\Lambda$ is obtained by transposing it and replacing $\lambda_{i}$ with $1 / \lambda_{i} . \Delta \sigma$ is obtained as

$$
\Delta \boldsymbol{\sigma}=\mathbf{V} \boldsymbol{\Lambda}^{-1} \mathbf{U}^{T} \Delta \mathbf{b}_{c} .
$$

The matrix multiplication can also be written in summation form as

$$
\Delta \boldsymbol{\sigma}=\sum_{i=1}^{n} \lambda_{i}^{-1} \mathbf{v}_{i}\left(\mathbf{u}_{i}\right)^{T} \Delta \mathbf{b}_{c}
$$

where $\mathbf{u}_{i}$ and $\mathbf{v}_{i}$ are the columns of matrices $\mathbf{U}$ and $\mathbf{V}$. In this equation, $\Delta \boldsymbol{\sigma}$ is the weighted sum of vectors $\mathbf{v}_{i}$. Therefore, an image is constructed by linear combinations of columns of $\mathbf{V}$. In this summation, if there exists some $\lambda_{i}$ very close to zero, and if the measurements are noisy, 
then some values of $\boldsymbol{\Lambda}^{-1}$ will grow drastically and cause errors in the reconstructed image. In order to prevent this, singular vectors corresponding to small singular values are truncated at $t<n$ and the upper limit in the above summation is set to $t$. The optimum truncation level depends on the object and the noise level. A method for the selection of the optimum truncation level is given in Gençer and Williamson (1998). In this method, the truncation level is determined such that $20 \log \left(\lambda_{1} / \lambda_{t}\right)=S N R$, where $\lambda_{t}$ is the singular value at the truncation level. Truncated SVD solves equation (11) in the least squares sense using only the retained image eigenvectors.

The above procedure can be applied iteratively in order to obtain an inverse for the forward transformation $\mathbf{F}_{c}$. However, it is not proved if this method will yield the correct image. To illustrate this point for a special case, consider two conductivity distributions $\sigma$ and $k \sigma$, where $k$ is a positive scaling factor. Both of these conductivity distributions will yield the same magnetic flux density measurements under the same Neumann boundary conditions. Therefore, at best we shall obtain a scaled version of the true conductivity by applying the above explained reconstruction algorithm.

Since a general proof for the uniqueness of the solution is not available at this stage, it is decided to at least scale the conductivity distribution found at each iteration using peripheral voltage measurements by solving the minimization problem (Birgül et al 2003):

$$
R=\min _{k}\left\|\frac{1}{k} \phi_{c}-\phi_{m}\right\|
$$

where $\phi_{m}$ and $\phi_{c}$ are the measured and calculated electrode potential values.

Steps of the overall iterative algorithm that uses sensitivity matrix and scaling principle are as follows (explained for two injected current patterns for simplicity):

(i) assume an initial conductivity distribution, $\sigma_{\text {ini }}$

(ii) calculate the combined sensitivity matrix $\mathbf{S}_{c}$ and magnetic flux density $\mathbf{b}_{i_{\text {ini }}}, i=1,2$;

(iii) compute

$$
\Delta \mathbf{b}_{c}=\mathbf{b}_{c_{\text {meas }}}-\mathbf{b}_{c_{\text {ini }}}
$$

where $\Delta \mathbf{b}_{c_{\text {meas }}}=\left[\begin{array}{l}\mathbf{b}_{1 \text { meas }} \\ \mathbf{b}_{2 \text { meas }}\end{array}\right]$ and $\Delta \mathbf{b}_{c_{\text {ini }}}=\left[\begin{array}{l}\mathbf{b}_{\text {1ini }} \\ \mathbf{b}_{2 \text { ini }}\end{array}\right]$;

(iv) calculate $\Delta \sigma$ by solving the combined sensitivity matrix equation and obtain the reconstructed conductivity $\sigma_{r}=\sigma_{\text {ini }}+\Delta \sigma_{\text {ini }}$;

(v) find electrode potential values $\phi_{r}$ corresponding to $\sigma_{r}$;

(vi) find $k$ using (15) and obtain the scaled conductivity $\sigma_{r s}=k \sigma_{r}$.

The algorithm can be iterated by replacing $\sigma_{\text {ini }}$ in step (i) with the scaled conductivity $\sigma_{r s}$.

\subsection{Error definitions}

In this study, two different error values are calculated. First a definition is given by $L 2$ norm of relative pixel errors and is defined as

$$
\varepsilon_{\sigma}=\sqrt{\frac{1}{N} \sum_{n=1}^{N} \frac{\left(\sigma_{t, n}-\sigma_{r s, n}\right)^{2}}{\sigma_{t, n}^{2}}} \times 100 \%
$$

where $\sigma_{t, n}$ and $\sigma_{r s, n}$ are the true and reconstructed conductivity values for the $n$th element. This error can be calculated over all elements $(N$ is the total number of elements in the FE model) or over elements corresponding to a subregion ( $N$ is the total number of elements in 
the desired region). Note that this error formula cannot be used for phantoms which have insulating regions.

The phantoms are composed of two separate zones which are either saline or sponge immersed in saline. These zones are expected to have uniform conductivity within themselves. In order to find to what extent these zonal conductivities are found correctly, another error criterion, namely average conductivity error, is also used. The average conductivity error is calculated as follows:

$$
\varepsilon_{\sigma, \mathrm{av}}=\frac{\left|\sigma_{t}-\frac{1}{M} \sum_{n=1}^{M} \sigma_{r s, n}\right|}{\sigma_{t}} \times 100 \%
$$

where $i=1-M$ are the indices of the conductivity pixels in the zone of interest. Average conductivity error reflects the bias in the zonal conductivity irrespective of the variance of the reconstruction noise.

\section{Results}

Several images are reconstructed for different conductivity distributions using inner magnetic flux density and peripheral voltage measurements. Although magnetic flux density is measured throughout the entire imaging region, measurements near the boundary, especially close to current injection electrodes, contain large errors. Measurements close to the boundary are not included in image reconstruction, i.e. they are not included in $\mathbf{b}_{c}$.

The magnitude of the bipolar dc current pulse is set to $25 \mathrm{~mA}$ and total duration of current $\left(T_{\mathrm{C}}\right)$ is equal to $42 \mathrm{~ms}$. The repetition time $\left(T_{\mathrm{R}}\right)$ and echo time $\left(T_{\mathrm{E}}\right)$ of the spin echo pulse sequence are $500 \mathrm{~ms}$ and $45 \mathrm{~ms}$. When 8 averages and 128 phase encoding gradients are used, the data acquisition time is $8 \mathrm{~min}$ for each experiment. Since two experiments (one with current injection and one without current) are required to reconstruct a conductivity image, the total data acquisition time needed is $16 \mathrm{~min}$. ac current with $1 \mathrm{kHz}$ frequency and $10 \mathrm{~mA}$ (rms) amplitude is used for EIT measurements. The magnetic flux density is measured on a $30 \times 30$ grid and each pixel is $2 \mathrm{~mm} \times 2 \mathrm{~mm}$. Two pixels at each side are excluded in image reconstruction. In other words, magnetic flux density on a $26 \times 26$ grid is used in reconstruction. Note that, although measurements from boundary are not used in the algorithm, conductivity is calculated for the entire imaging region on a $30 \times 30$ grid. The images for measured magnetic flux density and reconstructed conductivity presented throughout this section are therefore for $5.6 \mathrm{~cm} \times 5.6 \mathrm{~cm}$ and $6 \mathrm{~cm} \times 6 \mathrm{~cm}$ regions respectively.

The finite element mesh used in the calculation of the forward problem and sensitivity matrix calculation contains 961 nodes and 1800 first order triangular elements. Two triangular elements correspond to a pixel in the MRI grid. The sensitivity matrix is calculated for a uniform initial conductivity distribution of $20 \mathrm{mS} \mathrm{cm}^{-1}$.

Image reconstruction using the method in this paper requires the inversion of equation (9) where $\Delta \mathbf{b}$ is the measurement vector. The SNR of this vector determines the optimum truncation level. To calculate the SNR in $\Delta \mathbf{b}$ for a given object, the following formula is used:

$$
S N R=20 \log \frac{\left\|\mathbf{b}_{\text {obj }, c}-\mathbf{b}_{\text {uni }, c}\right\|}{\left\|\mathbf{b}_{\text {uni }, m}-\mathbf{b}_{\text {uni }, c}\right\|}
$$

where $\mathbf{b}_{\mathrm{obj}, c}$ is the calculated magnetic flux density for the object, and $\mathbf{b}_{\text {uni,c }}$ and $\mathbf{b}_{\text {uni, } m}$ are the calculated and measured magnetic flux densities for the uniform conductivity case. Using this formula, the SNR is calculated to be between 5 and $15 \mathrm{~dB}$ for different object cases. For an average SNR around $10 \mathrm{~dB}$, the optimum truncation level is found using the method in Gençer and Williamson (1998), and the singular vectors corresponding to singular values that are less than $35 \%$ of the maximum singular value are truncated in image reconstruction. This 


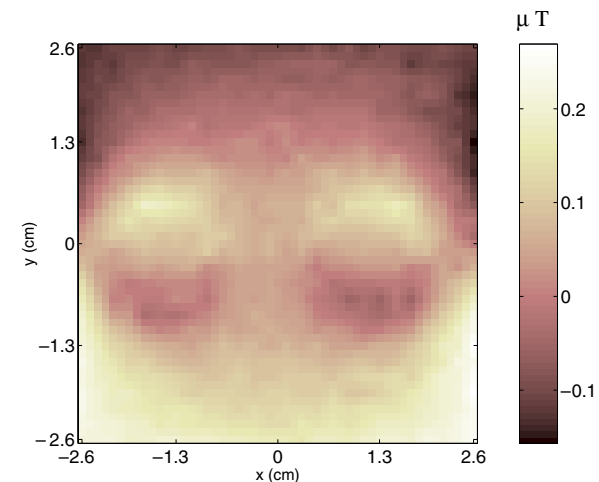

(a)

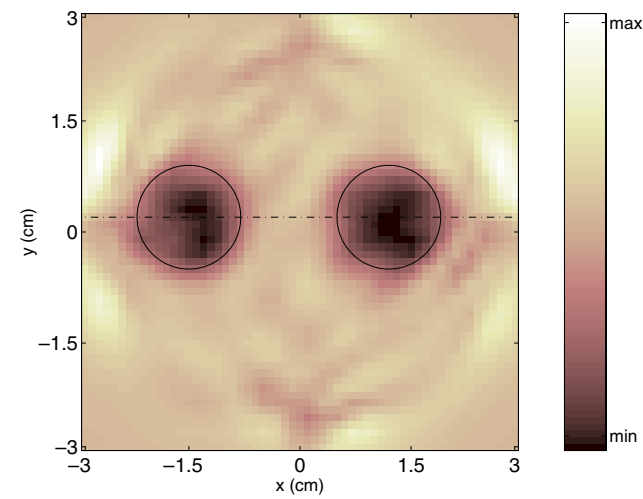

(c)

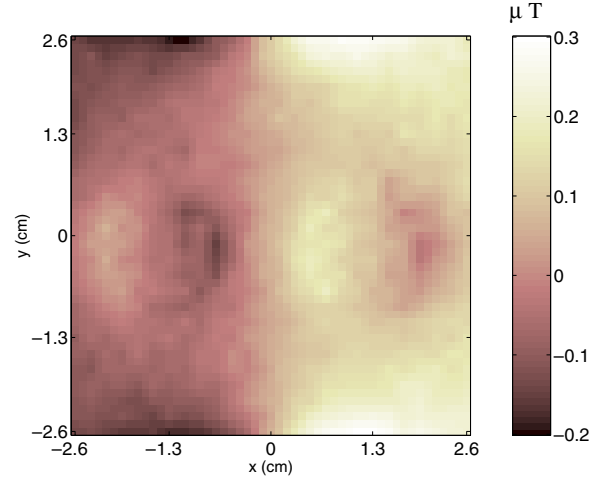

(b)

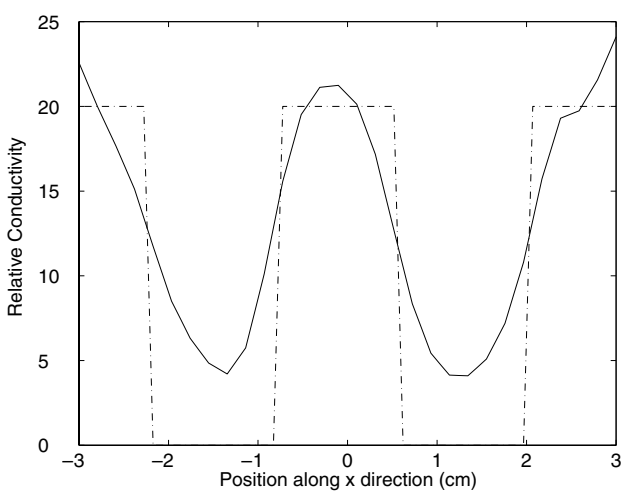

(d)

Figure 3. Two insulator objects of $1.5 \mathrm{~cm}$ diameter and placed $3 \mathrm{~cm}$ apart from each other where the distance is measured from the centres of the objects. (a) Magnetic flux density measurement for the horizontal current injection case. (b) Magnetic flux density for the vertical current injection case. (c) Reconstructed relative conductivity image when a truncation level of $0.35 \lambda_{\max }$ is used. (d) Profile images. The solid line shows the reconstructed and the dashed line shows the true profile images.

corresponds to using the first 158 basis vectors in image reconstruction. It is also empirically found that about 158 image vectors yield the best images.

Only a single iteration is made in reconstructing images using real data from the phantoms described below. We have observed that the algorithm has a divergent behaviour in the sense that the images degrade if two or more iterations are made.

\subsection{Insulator objects}

For insulator objects, relative conductivity images are reconstructed, i.e. voltage measurements for scaling are not used. Since the sensitivity matrix approach assumes low conductivity perturbations to be imaged, for insulator inhomogeneities quantitative accuracy of the reconstructed images is expected to be low. Therefore, only relative conductivity distributions are reconstructed here, to observe the edge response and position dependence of the method for high contrast objects. First, two circular objects with $1.5 \mathrm{~cm}$ diameter are placed $3 \mathrm{~cm}$ apart from each other where the distance is measured from the centres of the objects. In figures 3(a) and (b) magnetic flux density distributions for the horizontal and vertical current 


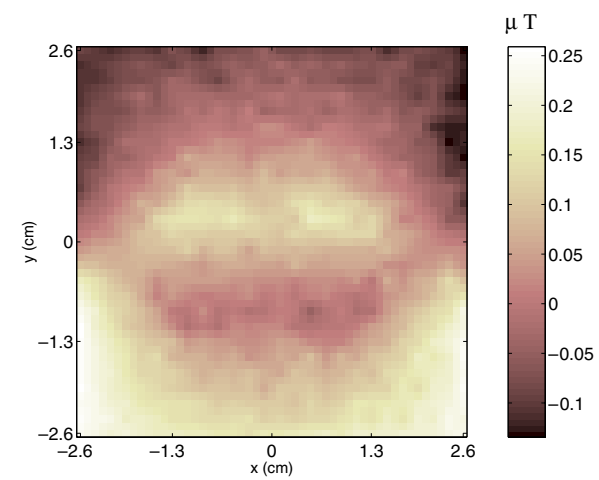

(a)

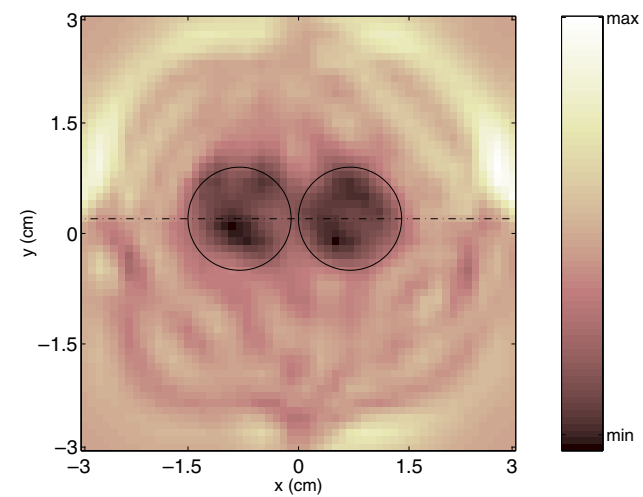

(c)

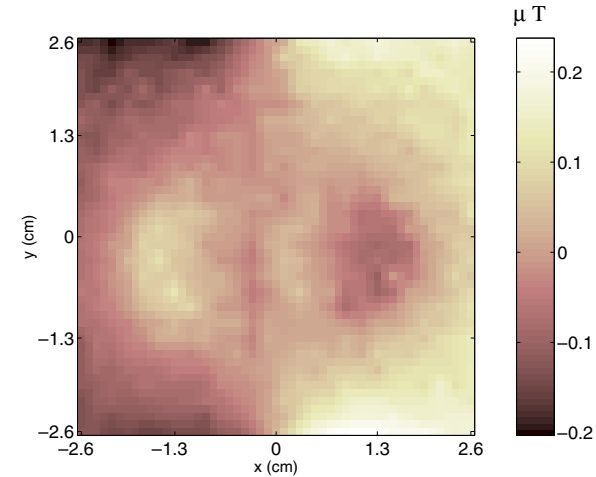

(b)

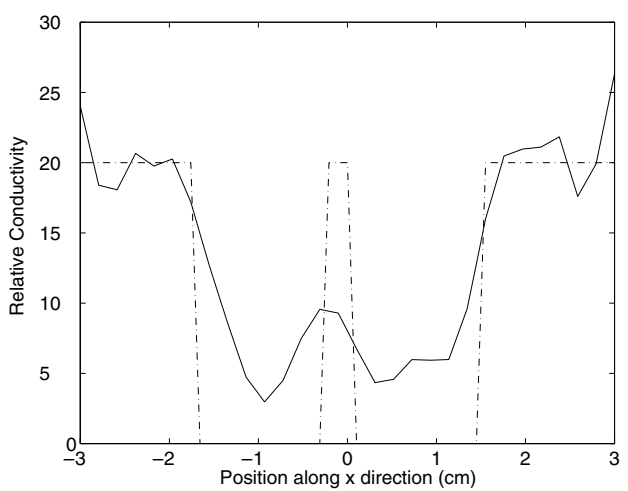

(d)

Figure 4. Two insulator objects of $1.5 \mathrm{~cm}$ diameter and placed $1.7 \mathrm{~cm}$ apart from each other where the distance is measured from the centres of the objects. (a) Magnetic flux density measurement for the horizontal current injection case. (b) Magnetic flux density for the vertical current injection case. (c) Reconstructed relative conductivity image when a truncation level of $0.35 \lambda_{\max }$ is used. (d) Image profile. The solid line shows the reconstructed and the dashed line shows the true profile images.

injection cases are presented. In figures 3(c) and (d), the reconstructed conductivity image and the image profile through the centre are presented respectively. Dashed lines show the true locations of the objects.

Next, the two circular insulator objects are brought closer and the distance between the centres is reduced to $1.7 \mathrm{~cm}$. This corresponds to one pixel separation between objects. The magnetic flux density measurements and the reconstructed conductivity distribution for this case are given in figure 4.

\subsection{Conducting objects}

Several experiments are carried out using conducting objects made from sponges immersed in the saline filled phantom. For these cases, both magnetic flux density and peripheral voltage values are measured. Note that voltage measurements are only used to scale the relative conductivity image to obtain the absolute conductivity values. In figure 5 , definitions for 


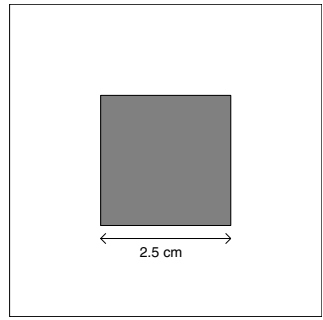

(a)

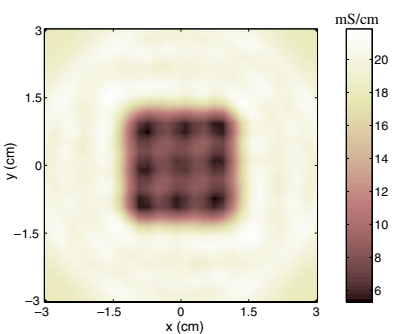

(d)

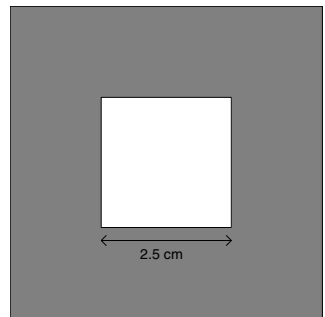

(b)

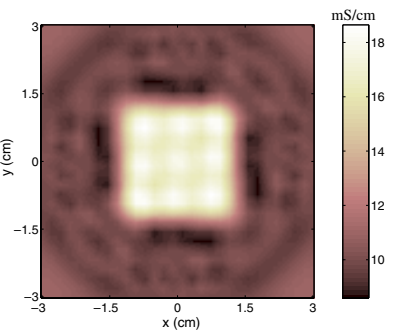

(e)

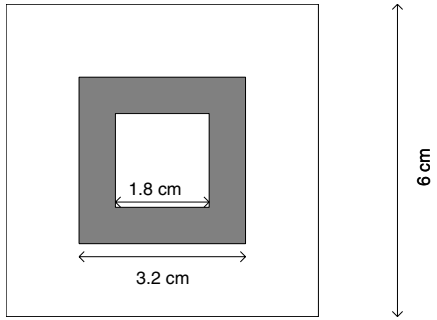

(c)

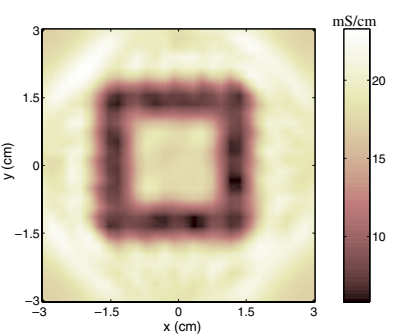

(f)

Figure 5. Definitions for the conducting objects. Grey regions indicate sponge that has conductivity around $10 \mathrm{mS} \mathrm{cm}$. White regions indicate saline solution with $20 \mathrm{mS} \mathrm{cm}^{-1}$ conductivity: (a) D1 case, (b) D2 case, (c) D3 case. Reconstructed conductivity images using noise-free simulated data and a truncation level of $0.35 \lambda_{\max }$ for (d) D1, (e) D2 and (f) D3.

Table 1. Average reconstructed conductivity values $\left(\mathrm{mS} \mathrm{cm}^{-1}\right)$ for the three conductor object cases. True, exp. and sim. stand for the true value, the reconstructed value using experimental data and the reconstructed value using simulation data.

\begin{tabular}{|c|c|c|c|c|c|c|c|c|c|}
\hline & \multicolumn{3}{|c|}{$D 1$} & \multicolumn{3}{|c|}{$D 2$} & \multicolumn{3}{|c|}{ D3 } \\
\hline & True & Exp. & Sim. & True & Exp. & Sim. & True & Exp. & Sim. \\
\hline Background & 20 & 19 & 19.8 & 10 & 11.6 & 10 & 20 & 20.8 & 19.3 \\
\hline Object & 10 & 14.7 & 8.3 & 20 & 16.5 & 16.6 & 10 & 15 & 9.5 \\
\hline
\end{tabular}

the conducting objects $D_{1}, D_{2}$ and $D_{3}$ are given. Sponge regions are shown in grey. In order to obtain the best conductivity image that can be reconstructed in one iteration and with the chosen truncation level, simulation studies are carried out. The reconstructed images for noise-free simulated data are presented in figures $5(\mathrm{~d})-(\mathrm{f})$. The saline conductivity and sponge region conductivity are taken as $20 \mathrm{mS} \mathrm{cm}{ }^{-1}$ and $10 \mathrm{mS} \mathrm{cm}^{-1}$ in simulations, respectively. The corresponding L2 norm of relative conductivity errors for the simulations is found to be $8 \%$, $7 \%, 11 \%$ for the entire images of D1, D2 and D3 cases, respectively. Average conductivity values for the saline and sponge regions are as given in table 1. In general, the background region's average conductivity regardless of whether it is saline or sponge is found to be within $4 \%$ error. However, the object region's conductivity has $17 \%, 17 \%$ and $5 \%$ error for the D1, D2 and D3 cases respectively.

After observing the best conductivity images that can be reconstructed for the selected truncation level with simulated noise-free data, the three cases are experimentally realized. For the $\mathrm{D} 1 \mathrm{case}$, a $2.5 \mathrm{~cm} \times 2.5 \mathrm{~cm}$ square object is placed at the centre of the imaging region. For this case, the object is less conductive compared to the background. Measured magnetic flux 


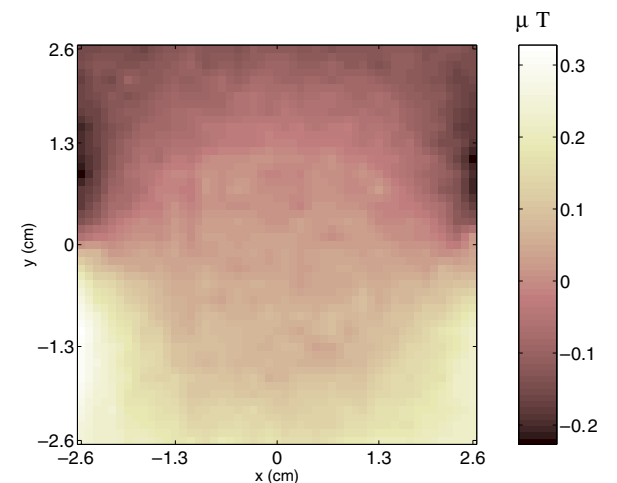

(a)

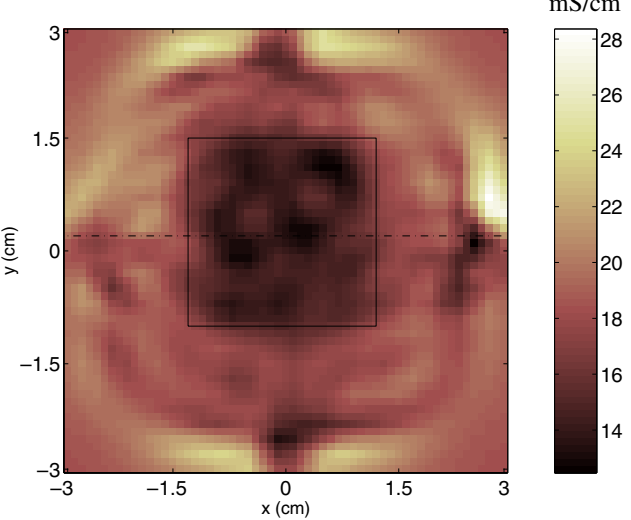

(c)

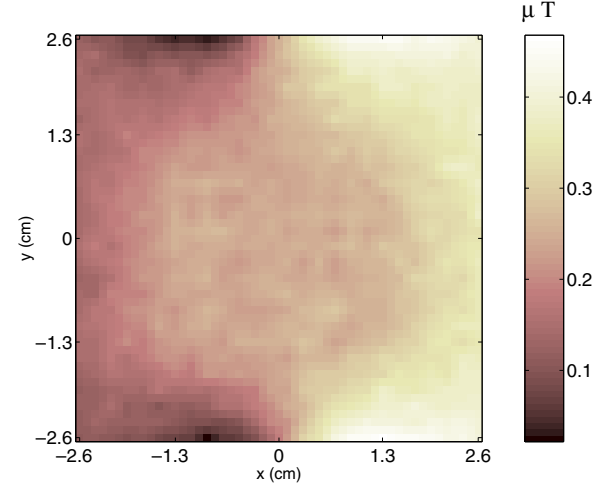

(b)

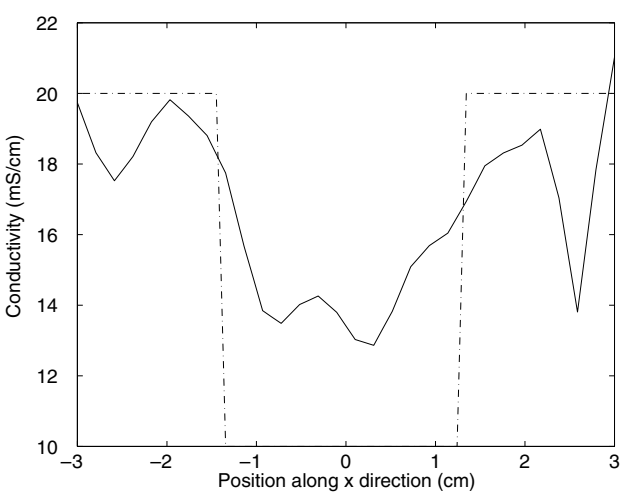

(d)

Figure 6. $2.5 \mathrm{~cm} \times 2.5 \mathrm{~cm}$ square object with conductivity $10 \mathrm{mS} \mathrm{cm} \mathrm{cm}^{-1}$ at the centre of the imaging region where background conductivity is $20 \mathrm{mS} \mathrm{cm}^{-1}$. (a) Magnetic flux density for the horizontal current injection case. (b) Magnetic flux density for the vertical current injection case. (c) Reconstructed conductivity image when a truncation level of $0.35 \lambda_{\max }$ is used. (d) Image profile. The solid line shows the reconstructed and the dashed line shows the true profile images.

density distributions and the reconstructed conductivity image are presented in figure 6 . The average reconstructed saline and sponge conductivity values from experimental data are found to be $19 \mathrm{mS} \mathrm{cm}^{-1}$ and $14.7 \mathrm{mS} \mathrm{cm}^{-1}$ respectively. The average conductivity of the sponge is found to be $47 \%$ higher than the value measured with the four-electrode measurement set-up (Baker 1989). In the four-electrode measurement set-up, the sponge fills the measurement cell and cannot expand freely. However, when we place a piece of sponge in our phantom, the sponge expands and its conductivity increases. Therefore, a higher conductivity value in the phantom experiments is expected. The error in average reconstructed conductivity for the saline region (background for this case) is found to be 5\%. For this region, the L2 norm of relative errors is $10 \%$.

In the next experiment (D2), the background and object conductivity values are switched by cutting out a $2.5 \mathrm{~cm} \times 2.5 \mathrm{~cm}$ square region from a $6 \mathrm{~cm} \times 6 \mathrm{~cm}$ sponge and placing it inside the phantom. In other words, background and object conductivity values are set to $10 \mathrm{mS} \mathrm{cm}{ }^{-1}$ (approximately) and $20 \mathrm{mS} \mathrm{cm}^{-1}$ respectively. The results for this case are given in figure 7 where average reconstructed background and object conductivity values are 


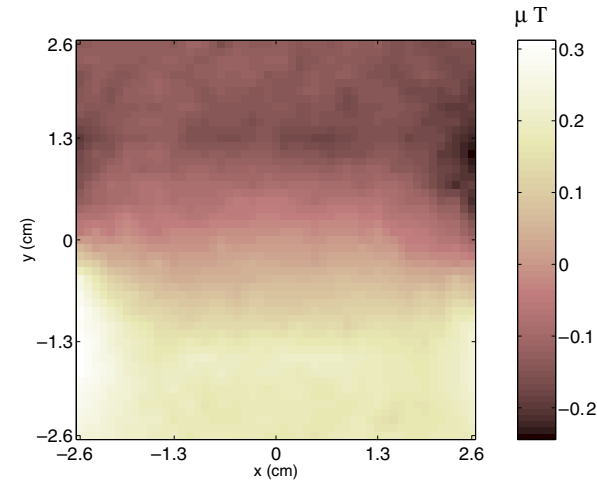

(a)

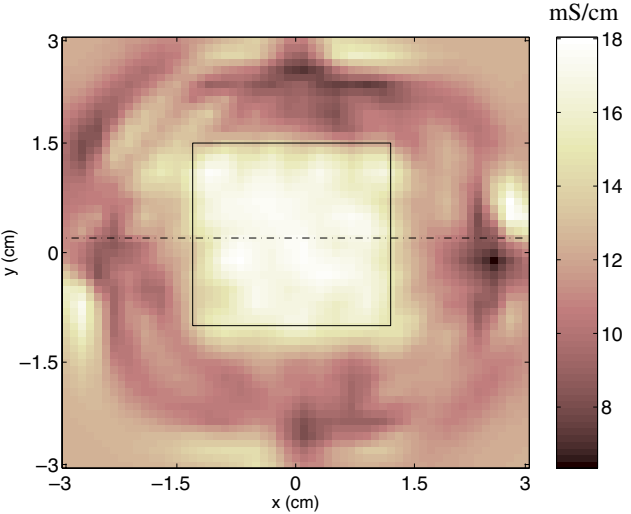

(c)

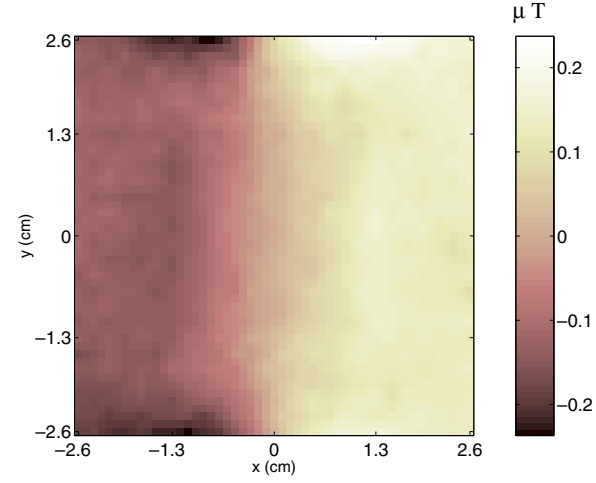

(b)

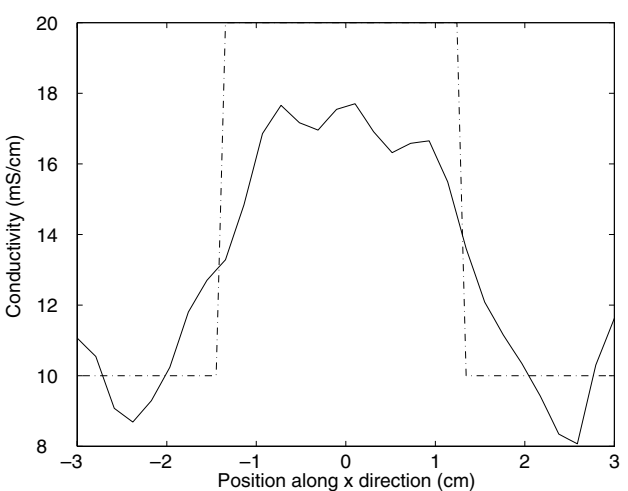

(d)

Figure 7. $2.5 \mathrm{~cm} \times 2.5 \mathrm{~cm}$ square object with conductivity $20 \mathrm{mS} \mathrm{cm}^{-1}$ at the centre of the imaging region where the background conductivity is $10 \mathrm{mS} \mathrm{cm}{ }^{-1}$. (a) Magnetic flux density for the horizontal current injection case. (b) Magnetic flux density for the vertical current injection case. (c) Reconstructed conductivity image when a truncation level of $0.35 \lambda_{\max }$ is used. (d) Image profile. The solid line shows the reconstructed and the dashed line shows the true profile images.

$11.1 \mathrm{mS} \mathrm{cm}^{-1}$ and $16.5 \mathrm{mS} \mathrm{cm}^{-1}$. Note that for this case, since sponge cannot expand freely, the reconstructed value is closer to that measured with the four-electrode measurement result. The average conductivity error in the saline region is $18 \%$ and the $\mathrm{L} 2$ norm of relative errors is $16 \%$ for this case.

Finally, a square ring object is placed at the centre of the imaging region for which background conductivity is $20 \mathrm{mS} \mathrm{cm}{ }^{-1}$ and object conductivity is around $10 \mathrm{mS} \mathrm{cm}-1$ (case D3). The definition for the object is given in figure 8 together with the magnetic flux density measurements and reconstructed conductivity distribution. The average reconstructed background conductivity is calculated to be $20.8 \mathrm{mS} \mathrm{cm} \mathrm{cm}^{-1}$ and the average reconstructed object conductivity is $15 \mathrm{mS} \mathrm{cm} \mathrm{cm}^{-1}$. The conductivity of the sponge region is close to that found for the D1 case. The average conductivity error in the saline region is calculated to be $4 \%$ and the $\mathrm{L} 2$ norm of relative errors is $10 \%$.

Reconstructed average conductivity values and L2 norm of relative errors are summarized in tables 1 and 2. For the D1, D2 and D3 cases, the L2 norms of relative errors over all pixels are found to be $13 \%, 17 \%$ and $14 \%$, respectively. 


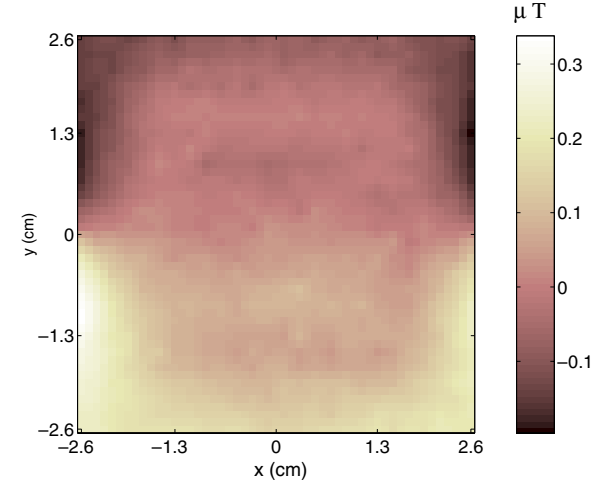

(a)

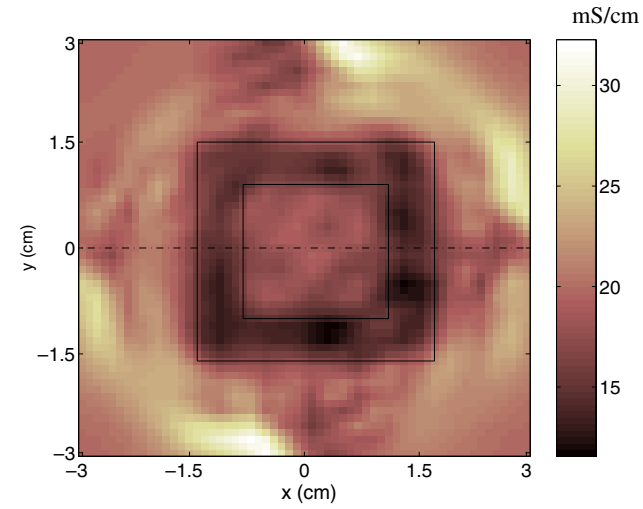

(c)

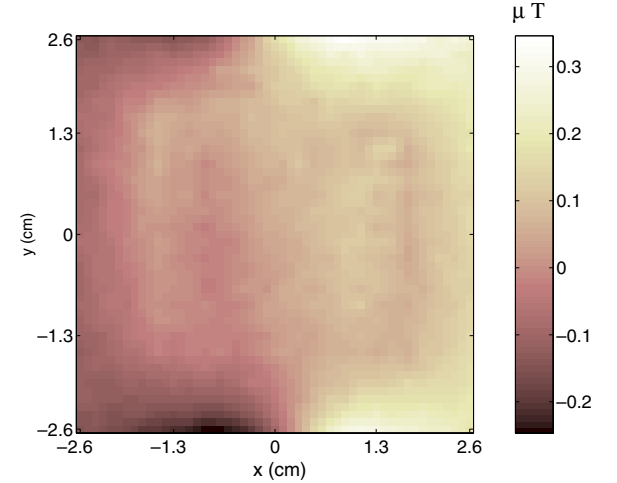

(b)

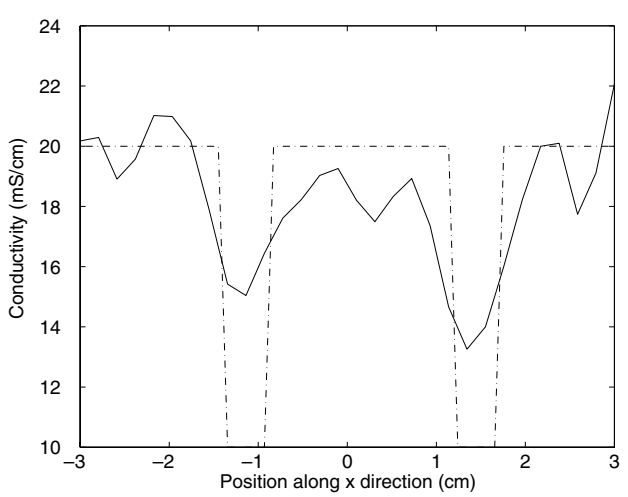

(d)

Figure 8. Ring object with conductivity $10 \mathrm{mS} \mathrm{cm}^{-1}$ placed at the centre of the imaging region where the background conductivity is $20 \mathrm{mS} \mathrm{cm}{ }^{-1}$. (a) Magnetic flux density for the horizontal current injection case. (b) Magnetic flux density for the vertical current injection case. (c) Reconstructed conductivity image when a truncation level of $0.35 \lambda_{\max }$ is used. (d) Image profile. The solid line shows the reconstructed and the dashed line shows the true profile images.

Table 2. L2 norms of relative error values (\%) calculated over all pixels.

\begin{tabular}{lrrr}
\hline & D1 & D2 & D3 \\
\hline Simulation & 8 & 7 & 11 \\
Experimental & 13 & 17 & 14 \\
\hline
\end{tabular}

\section{Discussion}

MR-EIT images reconstructed from experimental data are presented. Only one component of the magnetic flux density measured from a single slice is used to reconstruct images. For the experimental data, the L2 norm of relative errors in reconstructed conductivity images is found to be between 13 and $17 \%$. With simulated noise-free data, the L2 norm of relative conductivity errors is between 8 and $11 \%$. Errors in reconstruction with simulated data originate from the fact that only a single iteration is performed and that 
truncation is applied in the SVD procedure. The difference of the errors in experimental and simulated reconstructions can be attributed to low SNR and also to wrong estimation of sponge conductivity.

In a recent study by Lee et al (2003), relative $L^{2}$-errors of $25.5 \%$ and $32.3 \%$ with denoising and without denoising, respectively, were reported. We have not used any denoising in our reconstructions. The MR pixel size of their images is $0.6 \mathrm{~mm} \times 0.6 \mathrm{~mm}$ whereas our pixel size is $2 \mathrm{~mm} \times 2 \mathrm{~mm}$. Using larger pixel size results in higher SNR whereas spatial resolution is degraded. Therefore, sacrificing spatial resolution improves accuracy. Another way to improve the accuracy and the spatial resolution is increasing the amount of applied current. Increasing the injected current will result in higher magnetic flux density, hence larger SNR. However, the current cannot be increased without limit in medical applications. In fact, what determines the SNR and safe current limit is not the current at the electrode but the local current density. Birgül et al (2003) suggests that instead of using a single or double current injection profile with high current level at the electrodes, multiple current injection profiles with lower current amplitudes at different electrodes can be used to improve the quality of MR-EIT images. It is important to achieve a uniform current density distribution throughout the imaging to have uniform sensitivity distribution without exceeding the safety levels. In addition to optimizing the applied current pattern (Birgül et al 2003), improving MR system SNR given by Scott et al (1992), lower local current density values will be measurable. Medical device safety regulation, International Electrotechnical Commission (IEC)601-1 standard (1977), allows a patient auxiliary current up to $100 \mu \mathrm{A}$ in the frequency range $0.1 \mathrm{~Hz}-1 \mathrm{kHz}$. Since the currents used in MR-EIT based on the MRI pulse sequence developed by Scott et al (1991) are in this frequency range, for MR-EIT applications on human subjects a total injected current of $100 \mu \mathrm{A}$ must be aimed at.

It has also been observed that the quality of the reconstructed conductivity image degrades at the corners of the imaging region. The current density at the corners is less compared to that at other regions for both current injection cases and therefore the magnetic flux density is also less.

The effect of current carrying wires is excluded as mentioned in the experimental set-up section. It is difficult to model the lead wire geometry and surface currents on electrodes. In this study, only the magnetic flux density generated by the portions of the wires perpendicular to the $z$-direction is calculated and subtracted from measured $B_{z}$. This reduces the L2 norm of relative errors considerably. For example, for the D1 case, improvements in the L2 norm of relative errors are 3,1.2 and 6\% for all pixels, saline zone and sponge zone, respectively.

Although it is possible to obtain absolute conductivity values by scaling the image using only one peripheral voltage measurement, in this study 30 peripheral voltage measurements were used for scaling purpose. The only purpose for using many measurements is to improve the estimation of the scaling parameter, $k$. Determining $k$ using many voltage measurements by the least squares minimization method defined in equation (15) provides more immunity against measurement noise in peripheral voltages.

The purpose of this study is to investigate the experimental performance of MR-EIT with a sensitivity-based image reconstruction algorithm. An investigation of the spatial resolution which can be achieved by a sensitivity-based MR-EIT image reconstruction algorithm has been performed by İder and Birgül (1998) on simulated data, and full-width-at-half-maximum values smaller than a pixel size are reported. The simulation results given in this study are included to give an idea of the best conductivity image which can be reconstructed with noisefree data by using the chosen truncation level. A detailed simulation study is needed to study the effect of truncation level on spatial resolution. 
MR-EIT algorithms proposed to date assume isotropic conductivity values. It is very well known that anisotropy is certainly present in biological tissues. The error introduced as a result of assuming isotropy must be investigated if anisotropic conductivity distributions are to be imaged. Image reconstruction algorithms which take into account the anisotropic conductivity distribution should be developed.

Results given in figures 3 and 4 show that the locations of the high contrast (insulator) objects are found correctly and relative conductivity values in both cases are found to be almost the same. Furthermore, the edge response in both cases does not seem to be position dependent. As seen in figure 4, two high contrast objects which are only one pixel apart can be resolved.

The sensitivity matrix approach assumes that the changes in the conductivity and the corresponding changes in the magnetic flux density (or current density) are linearly related, around an initial conductivity distribution. This assumption is valid for small conductivity perturbations. For the cases where conductivity deviates more from initial conductivity, the error in reconstructed image will be higher. However, if the divergence problem, mentioned below, due to susceptibility artefacts under the electrodes can be solved, the iterative sensitivity matrix approach images will improve.

\subsection{Convergence of the algorithm}

The proposed algorithm is an iterative one where the sensitivity matrix is recalculated at each iteration. Although the algorithm converges for the noisy simulated data, it shows a divergent behaviour for the experimental case. For the noisy simulation case, the system noise is generated using the expression given by Scott et al (1992). However, in practice, in addition to the system noise, a susceptibility artifact is observed in magnetic flux density measurements under the electrode. This reduces the SNR and introduces errors around the electrode regions in the reconstructed conductivity images. In the iterative algorithm, the sensitivity matrix is to be regenerated with this conductivity distribution that contains errors in high sensitivity regions. The sensitivity matrix calculated with this conductivity distribution contains large errors which causes the divergence of the algorithm. Woo et al (2003) proposed the use of recessed electrodes to eliminate the which susceptibility artifact.

\subsection{Number of current injections}

The combined sensitivity matrix has a rank of 899 . This does not contradict our argument that conductivity can be found apart from a constant factor. In other words, since there is a single degree of freedom which cannot be determined from $B_{z}$ measurements only, we can at best expect the rank of the combined sensitivity matrix to be 899 . The fact that the rank is not less than 899 is also a very important observation indicating that apart from the constant factor, the sensitivity matrix approach determines conductivity uniquely. This conclusion is, of course, correct for small perturbations so that a single iteration is sufficient. The convergence and uniqueness of the full non-linear problem of finding the inverse of $\mathbf{F}$ is yet to be studied. It is also interesting to note that if only one current injection is used, the sensitivity matrix does not behave as well; its rank is 898 and furthermore the ratio $\lambda_{1} / \lambda_{898}=1300000$ whereas for the combined sensitivity matrix $\lambda_{1} / \lambda_{899}=500$. In other words, a single current injection is not enough to determine conductivity apart from a single degree of freedom. Furthermore with single current injection, the condition number of the remaining system is significantly smaller than that of the combined case. 


\section{3. $2 D$ versus $3 D$ calculation of magnetic flux density and sensitivity}

Phantoms that have been used in this study have finite thickness and therefore, calculation of magnetic field at the mid-planes of these phantoms requires a 3D FEM formulation by which effects of current flowing in all layers are more realistically incorporated. For this purpose $2 \mathrm{~cm}$ thick phantoms are divided into $201 \mathrm{~mm}$ thick slabs. The current is assumed to be divided among all slabs equally and $B_{z}$ is calculated using the Biot-Savart law at the mid-plane. For the specific phantoms used in this study, a significant improvement is not observed in the reconstructed images employing a sensitivity matrix derived from the 3D approach explained above. Since 2D FEM is computationally much less expensive than 3D FEM, the 2D formulation is used in this study. Several reasons why the 2D sensitivity gives comparable results to the $3 \mathrm{D}$ sensitivity may be the following:

(i) Correct 3D formulation of the forward problem requires detailed knowledge of the electrode impedance distribution at the electrode-phantom interface. Due to possible non-uniform distribution of electrode-phantom impedance, the current in the object may not be uniformly distributed along the thickness of the phantom. In practice, it appears that both 2D and 3D formulations are equally acceptable in modelling the real situation for the phantom geometry used in this study.

(ii) The slice thickness of the MRI images acquired in this study is $2 \mathrm{~cm}$. Therefore, the magnetic flux density obtained from the MRI phase images is an averaged version of the magnetic field along the thickness of the phantom. Consequently, a detailed 3D formulation of the forward solution is not really warranted.

\section{Conclusion}

In this study, the MR-EIT technique is implemented experimentally using the $0.15 \mathrm{~T}$ METU MRI system. The images presented in section 4 provide the first experimental validation of the sensitivity-based MR-EIT technique for conducting objects.

The reconstruction algorithm utilizes only one component of the current induced magnetic flux density from a single slice and is based on the relation between conductivity and magnetic flux density. Compared to algorithms that use the current density distribution calculated from magnetic flux density measurements, i.e. the type 1 algorithms, the proposed method has two advantages. First, no rotation of the object is required since only one component of the magnetic flux density is sufficient. This eliminates the problem in clinical realization of the method. The other advantage is that no derivative operation is used. For the algorithms based on current density distribution, the derivative of the magnetic flux density with respect to spatial coordinates is to be calculated to find the curl of the magnetic flux density measurements. This results in an algorithm which is highly sensitive to noise in magnetic flux density measurements. The ultimate spatial resolution is limited by the larger of the FEM element size and twice the MR image pixel size, when the curl operator is used (Birgül et al 2003). However, in the algorithm used in this study, no curl operator is required. On the other hand, spatial resolution is determined by the truncation level used for SVD inversion of the sensitivity matrix.

The results of this study show that conductivity images can be reconstructed with the L2 norm of relative error in conductivity values between $13 \%$ and $17 \%$, with experimental data for three different conductivity distributions.

Data acquisition times are of the order of minutes, similar to the durations of clinical MRI scans, therefore the imaging time is not expected to impose a problem in a clinical environment. Before the application of the technique to human subjects, the issues regarding the safe current levels and improved SNR must be resolved. 


\section{Acknowledgments}

This work is supported by Turkish Scientific and Technical Research Council (TÜBİTAK) Research Grant EEEAG-198006. The authors would like to thank Orçun Özbek and V Emre Arpinar of MRI Research Lab of METU for their efforts in data acquisition. This work is part of the PhD study of ÖB. BME and YZI are the thesis co-supervisors.

\section{References}

Baker L E 1989 Principles of impedance technique IEEE Eng. Med. Biol. Mag. 8 11-5

Birgül Ö 1997 Electrical impedance tomography using magnetic fields generated by injected currents MSc Thesis Dept of EE, Middle East Technical University

Birgül Ö 2002 Development of reconstruction algorithms for magnetic resonance-electrical impedance tomography and experimental realization PhD Thesis Dept of EE, Middle East Technical University

Birgül Ö, Eyüboğlu B M and İder Y Z 1999 Magnetic resonance-conductivity imaging Proc. IEEE EMBS Conf. (Atlanta, USA) p 1127

Birgül Ö, Eyüboğlu B M and İder Y Z 2001a New technique for high resolution absolute conductivity imaging using magnetic resonance-electrical impedance tomography (mr-eit) SPIE Physics of Medical Imaging 2001 vol 2 pp 880-8

Birgül Ö, Eyüboğlu B M and İder Y Z 2001b Magnetic resonance-conductivity imaging using 0.15 tesla MRI scanner Proc. IEEE EMBS Conf. CD-ROM

Birgül Ö, Eyüboğlu B M and İder Y Z 2003 Current constrained voltage scaled reconstruction (CCVSR) algorithm for magnetic resonance-electrical impedance tomography and performance with different probing current patterns Phys. Med. Biol. 48 653-71

Birgül Ö and İder Y Z 1995 Use of magnetic field generated by the internal distribution of injected currents for electrical impedance tomography Proc. IXth Int. Conf. on Bio-Impedance pp 418-9

Cherepenin V, Karpov A, Korjenevsky A, Kornienko, Mazaletskaya, Mazourov D and Meister D 2001 A 3D electrical impedance tomography (EIT) system for breast cancer detection Physiol. Meas. 22 9-18

Eyüboğlu B M and Özbek O 2003 Combined use of MRCDI and MR-EIT for lead field imaging 4th Conf. on Biomedical Applications of Electrical Impedance Tomography (UMIST, Manchester) p 51

Eyüboğlu B M, Reddy R and Leigh J S 2002 Magnetic resonance-electrical impedance tomography Patent no US6,397,095 B1 provisional patent application on 1 March 1999

Frerichs I 2000 Electrical impedance tomography (EIT) in applications related to lung and ventilation: a review of experimental and clinical activities Physiol. Meas. 21 R1-R21

Gamba R and Delpy D T 1997 Artefacts in mr images of electrical current distribution Phys. Med. Biol. 42 2481-91

Gençer N and Williamson S J 1998 Differential characterization of neural sources with the bimodal truncated SVD pseudo-inverse for EEG and MEG measurements IEEE Trans. Biomed. Eng. 45 827-38

Gonçalves S, de Munck J C, Heethaar R M, da Silva F H L and van Dijk B W 2000 The application of electrical impedance to reduce systematic errors in EEG inverse-problem-a simulation study Physiol. Meas. 21 379-93

İder Y Z and Birgül Ö 1998 Use of magnetic field generated by the internal distribution of injected currents for electrical impedance tomography (MR-EIT) Elektrik Turkish J. Elec. Eng. Comput. Sci. 6 591-604

İder Y Z and Müftüler 1997 Measurement of ac magnetic field distribution using magnetic resonance imaging IEEE Trans. Med. Imaging 16 617-22

İder Y Z, Onart S and Lionheart W R B 2003 Uniqueness and reconstruction in magnetic resonance-electrical impedance tomography(MR-EIT) Physiol. Meas. 24 215-25

International Electrotechnical Commission 1977 Safety of Medical Electronic Equipment: part-1. General requirements (601-1) Geneva, IEC

Khang H S, Lee B I, Oh S H, Woo E J, Lee S Y, Cho M H, Kwon O, Yoon J R and Seo J K 2002 J-substitution algorithm in magnetic resonance electrical impedance tomography (mreit): phantom experiments for static resistivity images IEEE Trans. Med. Imaging 21 695-702

Kwon O, Lee J-Y and Yoon J R 2002a Equipotential line method for magnetic resonance electrical impedance tomography Inv. Problems 18 1089-100

Kwon O, Woo E J, Yoon J R and Seo J K 2002b Magnetic resonance electrical impedance tomography (mreit): simulation study of $j$-substitution algorithm IEEE Trans. Biomed. Eng. 49 160-7

Lee B I, Oh S H, Woo E J, Lee S Y, Cho M H, Kwon O, Seo J K and B W S 2003 Static resistivity image of a cubic saline phantom in magnetic resonance electrical impedance tomography (MREIT) Physiol. Meas. 24 579-89

Liang Z P 1996 A model-based method for phase unwrapping IEEE Trans. Med. Imaging 15 893-7 
Mueller J L, Isaacson D and Newell J C 2001 Reconstruction of conductivity changes due to ventillation and perfusion from EIT data collected on a rectangular array Physiol. Meas. 22 97-106

Piperno G, Frei E H and Moshitzky 1990 Breast cancer screening by impedance measurements Front. Med. Biol. Eng 2 111-7

Scott G C, Joy M L G, Armstrong R L and Hankelman R M 1991 Measurement of non-uniform current density by magnetic resonance IEEE Trans. Med. Imaging $10362-74$

Scott G C, Joy M L G, Armstrong R L and Hankelman R M 1992 Sensitivity of magnetic resonance current density imaging J. Magn. Reson. 97 235-54

Silvester P P and Ferrari R L 1996 Finite Elements for Electrical Engineers (Cambridge: Cambridge University Press)

Woo E J, Lee S Y and Mun C W 1994 Impedance tomography using internal current density distribution measured by nuclear magnetic resonance Proc. SPIE 2299 377-85

Woo E J, Lee B I, Oh S H, Lee S Y, Cho M H, Kwon O and Seo J K 2003 Three-dimensional forward solver for magnetic resonance electrical impedance tomography (MREIT) using recessed electrode 4th Conf. on Biomedical Applications of Electrical Impedance Tomography (UMIST, Manchester) p 49

Zhang N 1992 Electrical impedance tomography based on current density imaging MSc Thesis, Dept of EE, University of Toronto 